\title{
Introduction to "Efficient local updates for undirected graphical models" by F. Stingo, G. Marchetti
}

\author{
Colin Fox
}

Published online: 20 November 2014

(C) Springer Science+Business Media New York 2014

Interconnectivity in systems can be represented by undirected graphs, and so graphical models naturally arise in many hierarchical Bayesian models, such as the neighbourhood structure in a segmented Gaussian Markov random field, or patterns of shared gene families across genomes, or friendship networks in Facebook. The paper by Stingo and Marchetti is motivated by a problem of inferring the interaction network for proteins from expression data. Inference over graph structures presents significant difficulties, particularly for MCMC sampling, because normalising constants usually depend on graph structure in an intractable way. For example, naive calculation for the segmented GMRF requires evaluating a determinant of the state-dependent precision matrix (concentration matrix in the present paper), which is generally computationally prohibitive for anything other than artifi- cially simplified problems. For the sub-class of decomposable graphs, the computational cost is made feasible by graph-theoretic results that provide a direct calculation of Cholesky factors, or equivalently the triangular decomposition. Stingo and Marchetti nicely summarise the theory for decomposable graphs and also dynamic algorithms over that class, describing ways that auxiliary data structures can be updated efficiently when using local moves. Their main contribution is a proposal for extending this machinery to general graphs. They show that by assuming a linear regression form for the likelihood, an approximated analysis over the general graphical model becomes tractable. Estimates presented for a protein network are informative, indicating that this paper makes a contribution to a topical, though notoriously difficult, problem in Bayesian modelling.

C. Fox $(\bowtie)$

Department of Physics, University of Otago, Dunedin 9016,

New Zealand

e-mail: colin.fox@otago.ac.nz 